\title{
Die iibrigen Kindeslagen, die Behandlung der Geburt im Allgemeinen und bei den verschiedenen Kindeslagen.
}

\section{Aetiologie, Mechanismus und Prognose der Vorderhaupts-, Stirn- und Gesichtslagen.*)}

Gusserow unterscheidet 4 Schädellagen. Bei der ersten steht die kleine Fontanelle links vorn, bei der zweiten rechts vorn, bei der dritten rechts hinten, bei der vierten links hinten. Bei den Schädellagen, welche mit graden Zahlen benannt sind, der 2. und 4., verläuft also auch die Pfeilnaht in dem mit grader Zahl benannten 2. schrägen Durchmesser. Ebenso ist das Verhältnis der 1. und 3. Schädell. zum 1. schrägen Durchmesser. Steht die kleine Fontanelle links oder rechts, so rechnen wir diese Stellung zur 1. resp. 2. Schädellage. Andere Autoren nehmen nur 2 Schädellagen mit 2 Unterarten an, je nachdem die kleine Fontanelle vorn oder hinten steht. Sehr häufig findet man bei 1. Schädellage die kleine Fontanelle bereits im Beginn der Geburt links vorn, bei 2. dagegen rechts hinten (1. und 3. Schädellage nach Gusserow). Es beruht diese Erscheinung auf dem Gesetz der Schwere, welches die Schädellagen überhaupt bedingt und bei aufrechter Stellung der Frau die kleine Fontanelle nach links vorn, bei liegender dagegen nach rechts hinten bringt. Im Allgemeinen dreht sich die anfangs hinten stehende kleine Fontanelle im Verlauf der Geburt nach

*) Verf. hat es vorgezogen, diesem und den folgenden Kapiteln eine selbstständige Stellung anzuweisen und sie nicht in dem Abschnitt: „Physiologie der Geburt" unterzubringen, weil sie vielfach das Gebiet der Pathologie der Geburt streifen. 
vorn. Hin und wieder dreht sie sich aber noch mehr nach. hinten, die grosse Fontanelle tritt tiefer und nach vorn (Vorderhaupts-, Vorderscheitellage). Eine Vorderhaupts lage ist also nichts weiter, als eine 3. oder 4. Schädellage, die als solche verläuft. Hierbei wird, indem die Stirn sich an die Symphyse anstemmt, die grosse Fontanelle zuerst geboren, sodann schneiden Scheitel und Hinterhaupt über den Damm, and schliesslich wird die Stirn und das übrige Gesicht unter der Schamfuge hervorgetrieben.

Bei Stirnlagen tritt die Stirn, die anfangs seitlich stand, tiefer und nach vorn. Sie wird dann mitsammt den Augen zuerst geboren, indem sich der Oberkiefer an die Symphyse anstemmt; sodann tritt der Schädel über den Damm, Oberkiefer, Nund und Kinn kommen unter dem Schambogen hervor.

Bei Gesichtslagen tritt das Kinn tiefer und nach vorn, selbst wenn es im Beginn der Geburt hinten stand. Vorderer Mundwinkel und Kinn werden in der Schamspalte sichtbar und, indem sich der Hals an die Symphyse anstemmt, schneiden Nase, Augen, Stirn und Schädel über den Damm.

Man unterscheidet bei den Vorderhaupts-, Stirn- und Gesichtslagen, je nachdem der Rücken links oder rechts liegt, eine 1. und 2. Lage. Die 3 Lagen haben dieselbe Aetiologie, die mit Ausnahme von No.5 (s. S. 27) erst mit dem Beginn der Wehen sich geltend macht. Bei allen wird nämlich das Hinterhaupt zurückgehalten, und es hängt mehr von Zufälligkeiten ab, ob das Vorderhaupt oder die Stirn oder schliesslich das Gesicht tiefer tritt. Das Hinterhaupt wird zurückgehalten, weil es sich. am seitlichen Beckenrand anstemmt (No. 3 n. 1), oder weil die Hebelarme, in welche die Wirbelsäule die Schädelbasis teilt, (s. S. 23) gleich sind (No. 1 und 2). In letzterem Fall wird das eine Mal das Vorder-, das andere Mal das Hinterhaupt tiefer treten. Ist eimmal die Deflexionshaltung des Kopfes eingeleitet, so liegt der kürzere Hebelarm nach dem Vorderhaupt, der Stirn, dem Kinn hin und diese Teile treten dann tiefer, analog der 1. Drehung bei der 1. und 2. Schädellage. Der Teil aber, welcher tiefer tritt, wird durch die schiefe Ebene des Obturator int. (J.Veit) auch nach vorn dirigirt (2. Drehung). Die 3. Drehung berult darauf, dass Stirn resp. Oberkiefer 
resp. der Hals sich an die Symphyse anstemmen, und nun die nach vorn wirkende Druckkraft des Beckenbodens deu Schädel über den Damm wälzt.

Die speziellen Ursachen für die 3 Deflexionslagen sind folgende:

1) Angeborene Dolichocephalie (Gleichheit der

2) Rundkopfbildung bei kleinem Kopf $\}$ Hebelarme.)

3) Plattes Becken (das breite Hinterhaupt findet in der verengten Conjugata keinen Platz und weicht daher seitlich ab).

4) Dextro- oder Sinistroversion des Uterus.

Dieselbe bringt das Hinterhaupt zum Abweichen nach der entgegengesetzten Seite, also bei Dextroversion und 1. Schädellage nach der linken Darmbeinschanfel. Bei Dextroversion und 2. Schädellage hat man sich das Zustandekommen einer der 3 Deflexionslagen so erklärt, dass der Wehendruck das nach oben gerichtete Gesicht nach abwärts treibt.

5) Angeborne Geschwülste am Hals (Struma).

Diese bringen den Kopf schon von vornherein in eine. Gesichtslagenhaltung hinein.

Den 3 Lagen ist ferner der Umstand gemeinsam, dass bei ihnen grössere Durchmesser des kindlichen Schädels das Becken und die Schamspalte passiren müssen, und dass das breitere Hinterhaupt an der langen hinteren. Beckenwand herunter und über den Damm treten muss. Hieraus resultirt meistens (nicht bei kleinem Kopf) eine lüngere Dauer der Geburt, die für Mutter und Kind manche Gefabren mit sich bringt, und eine bedeutendere Gefährdung des Damms. Die Prognose der genannten 3 Lagen ist also im allgemeinen eine ungünstigere als die der normalen Schädellage.

Da die erste Gesichtslage sich aus der 1. Schädellage entwickelt, und bei dieser das Hinterhaupt gewöhnlich links vorn steht, so finden wir bei der 1. Gesichtslage das Kinn im Beginn der Geburt rechts hinten stehend. Bleibt das Kinn, wie das in extrem seltenen Fällen gesehieht, hinten stehen, so ist die spontane Geburt unmöglich! 
Die Stirnlage geht leicht in eine Gesichts- oder Vorderhauptslage über.

Die Geburtsgeschwulst sitzt auf dem vorangehenden Teil, Gegend der grossen Fontanelle, Stirn, vorderem Mundwinkel. Die Kopfform geht so charakteristische Veränderungen ein, dass man aus ihr noch nachträglich die Diagnose der betr. Lage stellen kann (Olshausen).

\section{Diagnose der verschiedenen Kopflagen.}

Die Diagnose: Kopflage ist bei nicht zu dioken und straffen Bauch- und Uteruswandungen schon durch die äussere Untersuchung zu stellen (s. S. 13), die deshalb der inneren Untersuchung stets vorauszugehen hat.

\section{Diagnose der Schädellagen.}

Bei der inneren Untersuchung, die bei geschlossenem $\mathrm{Mm}$. durch Palpation vom Scheidengewölbe aus angestellt wird, erkennt man den Schädel an seinen Nähten. Man setzt am besten den untersuchenden Finger hinter der Symphyse an den Schädel an und führt ihn dann soweit wie möglich nach hinten. Auf diesem Weg gelangt man, vorausgesetzt, dass der Kopf noch nicht im Beckenausgang steht, zu einer Naht, die entweder, bei 1. und 2. Schädellage, ein Schenkel der Lambdanaht, bei 3 . und 4. Schädellage ein Schenkel der Kranznaht, oder die Pfeilnaht selbst ist. Die beiden erstgenannten Nähte führen den Untersucher zur kleinen resp. grossen Fontanelle, die Pfeilnaht leitet den sie nach rechts und links abtastenden Finger zu beiden Fontanellen, die man lediglich daran mit Sicherheit erkennt, dass in ihnen 3 resp. 4 Nähte zusammenstossen.

Der Anfänger soll te erst dann die Diagnose einer bestimmten Schädellage mit Sicherheit aus sprechen, wenner beide Fontanellen mit Sicherheit gefühlt hat! Za dem Ende ist es oft zweckmässig, in der linken Beckenhälfte mit dem rechten Finger, in der rechten mit dem linken zu untersuchen. Sind die Fontanellen gefunden, so bestimmt man, welche tiefer steht, und wo die tiefer stehende Fontanelle im Verhältnis zur anderen und zum Becken sich befindet, ob vorn, hinten, rechts, links, rechts vorn, rechts hinten, links vorn, links hinten. 
Steht der Kopf bereits im Beckenausgang, so trifft der von der Symphyse nach hinten geführte Finger häufig direct auf die kleine Fontanelle und gelangt, indem er weiter nach hinten geht, an der Pfeilnaht entlang gleitend zur grossen Fontanelle.

\section{Diagnose der Stirnlage.}

Der von der Symphyse nach hinten geführte Finger trifft eine breite Knochenfläche, rechts oder links von derselben (bei 1. Stirnlage rechts), und zwar manchmal schon mehr nach vorn, findet der Finger die scharfen Supraorbitalränder und den Nasenrücken, nach der anderen Seite die grosse Fontanelle.

\section{Diagnose der Gesichtslage.}

Schon die äussere Untersuchung weist auf eine Gesichtslage hin, falls man z. B. bei 1. Kopflage die Herztöne auffallend deutlich weit rechts von der Lin. alba hört, und der Schädel über dem linken Schambeinast auffallend prominiert. Bei der inneren Untersuchung fühlt der nach hinten geführte Finger keine Naht, sondern unregelmässige Vertiefungen und Erhöhungen. Der nach den Seiten des Beckens hingeleitete Finger tastet an der einen Seite, (bei 1. Gesichtslage links vorn). die breite Knochenfläche der Stirn, die im Beginn der Geburt häufig tiefer steht, als das Kinn, nach der anderen Seite den Nasenrücken, die Nasenlöcher, die Querspalte des Mundes. (mit den dahinter gelegenen Kieferrändern), das Kinn.

Der Muad ist nur dann mit dem After zu verwechseln, wenn er durch eine starke Gesichtsgeschwulst eine runde rüsselfömige Gestalt angenommen hat. Trotzdem darf ein solcher diagnostischer Irrthum nicht vorkommen, weil man niemals auf ein einziges Zeichen hin die Diagnose einer bestimmten Lage stellen soll.

\section{Beckenendlagen.}

A etiologie: Missverhältnis zwischen der Grösse des Uterus und der Frucht (Häufigkeit bei unreifen, bei Zwillingsfrüchten, bei Hydramnion).

Die Beckenendlagen zerfallen in Steiss-, Fuss- und Knielagen. 
Fühlt man neben dem Steiss die Füsse, so spricht man von Steiss-Fusslage oder gemischter Steissgeburt.

Je nachdem der Rücken links oder rechts steht, unterscheidet man 1. und 2. Steiss-, resp. Knie-, resp. Fusslage. Die Fusslagen teilt man, je nachdem beide oder nur der vordere Fuss herabgesunken sind, in vollkommene und unvollkommene Fusslagen ein.

\section{Geburtsmechanismus der Beckenendlagen.}

Bei Fuss- und Knielagen werden diese Teile ohne besonderen Mechanismus geboren. Nur wenn bei unvollkommener Fusslage der hintere Fuss vorliegt, dreht sich derselbe nach vorn, weil das emporgeschlagene Bein mitsamt der Hüfte in der Kreuzbeinaushöhlung mehr Platz findet. Hierbei dreht sich natürlich auch der Rücken nach der anderen Seite, so dass aus einer 1. Fusslage eine 2. wird.

Bei 1. Steisslage tritt die vordere linke Hüfte tiefer und von rechts ber nach vorn, die Hüftbreite dreht sich also aus dem 2 schrägen des Beckeneingangs in oder annähernd in den geraden des Beckenausgangs. (Der Grund der Drehung ist derselbe wie bei Schädellage.)

Die vordere Hüfte wird zuerst geboren und stemmt sich zu gleicher Zeit an die Sympbyse an, die hintere Hüfte schneidet über den Damm.

Der Rumpf tritt gewöhnlich im 2. schrägen Durchmesser ein und aus, so dass der Rücken des Kindes nach links und vorn gerichtet ist. Die Schulterbreite dagegen tritt im 2. schrägen ein und im graden aus. Mitsamt dem Rumpf werden die Arme in ihrer natürlichen Haltung geboren.

Nach Geburt des Rumpfes dreht sich der Rücken nach vorn, indem der Kopf (wie bei Schädell.) aus dem queren oder 1. schrägen Durchmesser des Beckeneingangs in den graden des Beckenausgangs tritt. Das Hinterhaupt stemmt sich an die Symphyse an, und der Kopf wird in seiner natürlichen Haltung, d. h. mit auf die Brust geneigtem Kinn, über den Damm geboren.

\section{Diagnose der Steisslagen.}

Bei der äusseren Untersuchung fühlt man den härteren und leichter ballotirenden Teil im Fundus, bei der inneren 
fühlt man, von vorn uach hinten gehend, den $A$ nus, über ihm die Processus spinosi des Kreuzbeins, neben ihm beide oder nur das mehr nach vorn gerichtete Tuber ischii. Die Geschlechtsteile sind häufig gar nicht zu fühlen.

\section{Diagnose der Fusslagen.}

Bei der äusseren Untersuchung imponiert die Fusslage manchmal als Querlage, indem der Steiss zunächst auf der Darmbeinschaufel aufsteht. Bei der inneren Untersuchung wird dieser Verdacht noch bestärkt, weil bei stehender Blase die Füsse nicht immer zu fühlen sind. Dieser diagnostiscbe Irrthum, der häufig gemacht wird, hat nichts zu bedeuten; denu der Arzt wird bei völlig erweitertem Muttermund die Blase sprengen, dann sofort einen Fuss erreichen und der Umgebung durch die rasch ansgefübrte Wendung imponieren. Den Fuss erkennt man bei der inneren Untersuchung an der Ferse und unterscheidet ihn weiter von der Hand durch das Fehlen des Daumens, durch die Kürze der Zehen. Welcher Fuss vorliegt, erkennt man daran, dass man die Zehen nach vorn dreht. Beim rechten Fuss muss man nun vom Innenrand nach rechts gehen, um zum Aussenrand zu kommen, beim linken nach links (d. h. nach der rechten resp. linken Seite der Mutter).

\section{Diagnose der Knielagen.}

Das Knie kann mit dem Ellenbogen verwechselt werden. Indessen erreicht man vom Knie aus entweder den Steiss oder den Fuss, vom Ellenbogen aus die Schulter oder die Hand.

\section{Querlagen.}

Aetiologie:

1) Hängebauch: Der Fundus fällt stark nach vorn, der Kopf weicht infolgedessen vom Becken ab.

2) Enges Becken: Der Kopf weicht vom Beckeneingang ab, weil er auf demselben keinen Platz findet.

3) Kugelform oder Schlaff heit der Gebärmutter: Der Foetus kann ohne von Seiten der Uteruswand Widerstand zu finden, jede beliebige Lage einnehmen (Zwillingsgeburt, Fiydramnion oder Placenta praevia).

Da Schlaffheit der Gebärmutter und Hängebauch sich in der Regel nur bei Mehrgebärenden finden, so kommen Quer- 
lagen bei diesen viel häufiger vor. Querlage bei einer I p. weist. auf ein enges Becken hin, falls nicht etwa Zwillingsgeburt oder Hydramnion oder Placenta praevia bei derselben vorliegt.

\section{Geburtsmechanismus bei Querlagen.}

Eine ausgetragene und lebende Frucht kann bei Querlage nicht spontan geboren werden. Die spontane Geburt durch "Selbstentwicklung" oder durch die "Entwicklung conduplicato corpore" kommt nur bei unreifen oder ausgetragenen aber toten Kindern vor. Auch in letzterem Fall ist die Mutter der Gefahr der Zerreissung der Gebärmutter in hohem Masse ausgesetzt, der sie im Allgemeinen bei jeder Querlage erliegt, falls sie nicht etwa vorher an Sepsis zu Grunde geht. Die Selbstentwicklung besteht darin, dass der Steiss des Kindes neben der Schulter vorbei durchs Becken hindurch getrieben wird, die Geburt conduplicato corpore in der Geburt der Schulter, dann der gleichzeitigen Geburt von Kopf und Bauch.

\section{Diagnose der Querlagen.}

Bei der äusseren Untersuchung fühlt man rechts und links je einen grossen Teil, der Beckeneingang ist leer, die Herztöne sind in der Nabelgegend hörbar. Bei der inneren Untersu chung fühlt man die Schulter, die man an der dreieckigen Scapula und der länglichen dünnen Clavicula erkennt. Aus dem Verhältnis dieser beiden Gebilde zur vorderen Beckenwand erkennt man ferner, ob der Bauch oder der Rücken vorne liegt. Der Schluss der Achselhöhle giebt die Richtung, in welcher der Kopf liegt. Ehe man eine bestimmte Diagnose ausspricht, mus man sowohl die Clavicula als auch die Scapula gefühlt haben. Kine vollständige Diagnose würde z. B. lauten: Querlage, Kopf links, Rücken nach vorn, (1. Querlage, 1. Unterart) oder Kopf rechts, Bauch nach vor», (2. Querlage 2. Unterart).

Bei Querlagen fällt sehr häufig der untere Arm vor. Indessen ist Armvorfall an sich kein sicheres Zeichen der Querlage, da er auch bei Schädellage vorkommt. Zur DifferentialDiagnose muss man den Arm nach oben verfolgen:

Man stösst dabei entweder auf die Schulter oder den Kopf. Ist man zweifelhaft, ob man es mit einer Querlage oder 
Steisslage zu thun hat, so hole man die vorliegende Extremität herunter.

\section{Die geburtshülfliche Untersuchung und die Behandlung der normalen Geburt bei 1. u. 2. Schädellage.}

Bei der inneren Untersuchung einer Kreissenden kann man durch unreine Finger oder Instrumente, die am Muttermund vorhandenen frischen Wunden inficiren und hierdurch den Tod einer Frau herbeiführen, die ohne innere Untersuchung sicher am Leben geblieben wäre. Man muss daher als obersten Grundsatz der Geburtshülfe das: "Nil nocere" aufstellen. Die Erreichung dieses Ziels ist nur durch peinlichste Antisepsis und Reinlichkeit möglich. Von diesem Gesichtspunkt ausgehend, muss der Arzt oder Praktikant zu jeder Geburt folgende Gegenstände mit sich nehmen: Eine grössere Quantität reiner Carbolsäure $(200 \mathrm{~g})$, Sublimatpastillen mit einem Gehait von $1 \mathrm{~g}$ Sublimat, Keine sogenannte Wurzelbürste, $5 \%$ iges Carbolvaseline resp. Boroglycerinlanolin*), einen Heberschlauch mit Glasrohr, einen Messcylinder für $30 \mathrm{~g}$, ein Packet Salicylwatte und ein Packet 10-20\% ige Jodoformgaze; ferner einen neusilbernen männlichen und einen Trachealkatheter, ein Thermometer und ein Stethoscop. Der Praktikant bringt liese Gegenstände in einer leinenen Tasche (die ausgekocht werden kann) unter, der Arzt nimmt am besten seine grosse Tasche mit, in der sich neben sonstigen Instrumenten die genannten Dinge finden sollten (s. geb. Instrumentarium). Nichts ist unangenehmer als wenn man, bei der Kreissenden angelangt, irgend ein notwendiges Instrument nicht zur Hand hat.

Niemals benutze der Arzt oder Praktikant die Utensilien der Hebamme, speciell ersetze man den Irrigator, falls er nicht

*) Das Boroglycerinlanolin, welches unter dem Namen Dr. Graf'sches Boroglycerin neuerdings in den Handel gekommen ist, kan்n Verf. aufs wärmste empfehlen. Dasselbe ist verpackt in Zinntuben, so dass eine nachträgliche Verunreinigung des keimfreien Präparats nicht möglich ist. Ausserdem ist das genannte Mittel von ausgezeichneter Wirkung gegen die durch die verschiedene Antiseptica erzengte Sprödigkeit der Haut.

D ̈̈ hrssen, Geburtshülf liches Vademecum. 
ganz neu ist, durch einen beliebigen 2 Liter fassenden Topf, in den man den Gummischlanch, den sogenannten Saugheber, hineinhängt. Für den tadellosen Zustand seiner Utensilien muss der Arzt einstehen können, für die der Hebamme kann er es nicht. Ausserdem gebrauche man die antiseptischen Lösungen der Hebammen deswegen nicht, weil die Hebamme in der Praxis pauperum dieselben selbst bezahlen muss. Nebenbei sei noch bemerkt, dass den preussischen Hebammen nur der Gebrauch der $3 \%$ igen Carbolsäure gestattet ist. Bei der Kreissenden angelangt, lege der Arztzunächst ein 'Thermometer ein. Die wichtigsten und häufigsten Störungen der Geburt, die stattgehabte septische Infection resp. die Zersetzung des Uterusinhalts und die drohende Uterusruptur zeigen sich zuerst durch Temperaturerhöhung an. Besonders wichtig wird die Temperaturmessung in Fällen, wo die Geburt, sich nach dem Blasensprung in die Länge gezogen hat, wo vielleicht schon Entbindungsversuche stattgehabt haben, bei Aborten. Stirbt eine Frau an Puerperalfieber, so fällt die Schuld nicht auf den Arzt, wenn vor seiner ersten inneren Untersuchung die Kreissende bereits fieberte. Auf die betreffende Hebamme lässt sich heutzutage nicht mohr ohne Weiteres die Schuld an einem septischen Todesfall schieben, wenn es sich um eine in streng antiseptischer Schule gebildete Hebamme handelt. Diese desinficirt sich viel strenger als mancher Arzt und wird sich deswegen auch nicht ohne Protest die Schuld an einem unglücklichen Fall aufbürden lassen.

Die Zählung des Pulses nimmt man besser erst später vor, wenn die Aufregung der Kreissenden über die Ankunft des Arztes sich gelegt hat.

Falls man nicht schon auf dem Weg zur Geburt über diese Punkte aufgeklärt ist, erkundige man sich nach dem Alter der Kreissenden. der Dauer der Geburt, dem Zeitpunkt des Blasensprunges, nach der Beschaffenheit der Wehen, nach der letzten Menstruation, nach der Zahl der Geburten und der etwa geleisteten Kunsthülfe, sowie ihrem Resultat. Bei ungünstigem Verlauf ist anf Rachitis zu inquiriren und das Becken zu messen. Von hereditären Krankheiten oder Krankheit:anlagen braucht man nur auf Phthise zu fahnden, die das 
Stillen verbietet. Die Frage, ob die Kreissende an Syphilis oder Gonorrhoe leidet, wird man erst in Folge bestimmter lokaler Befunde aufwerfen. $\mathrm{Da}$ die Frau in der Regel von ihrem eigenen Mann angesteckt ist, meistens aber keine Ahnung davon hat, so muss der Arzt hier sehr vorsichtig vorgehen, um nicht das eheliche Glück zu zerstören.

Die äussere Untersuchung hat vor Allem die Lage des Kindes und die Frequenz der kindlichen Herztöne zu berücksichtigen. Diese beiden Punkte können nur während der Wehenpause und bei grosser Unruhe der Kreissenden manchmal erst nach Einathmung einiger Tropfen Chloroform festgestellt werden. Während der Wehe dagegen tritt der Contractionsring für das Gefühl deutlicher hervor. Eine stärkere Anfüllung der Blase, die sich als rundlicher praller Tumor über der Symphyse dokumentiert, darf nicht übersehen und muss, da sie den Fortschritt der Geburt ernstlich hemmen kann, sofort, ev. durch den Katheterismus beseitigt werden. Die spontane Entleerung gelingt häufig deswegen nicht mehr, weil der Kopf die Urethra stark gegen die Symphyse andrückt.

Bei Beginn der äusseren Untersuchung gibt man der Hebamme den Auftrag, für 2-3 Schüsseln und einen 1-2 Liter fassenden Topf mit warmem Wasser, für eine reine Schürze, reine Handtücher, reine Unterlagen, für ein Stechbecken und einen reinen Eimer zu sorgen.

\section{Desinfektion des Geburtshelfers.}

Nach beendeter äusserer Untersuchung streift sich der Geburtshelfer nach Ablegung des Rocks und Anlegung einer reinen Schürze oder noch besser eines Operationsmantels die Hemdsärmel bis über den Ellenbogen in die Höhe, zieht alle Ringe ab, reinigt die Nägel vom sichtbaren Schmutz und bürstet die Nägel, die Hände (jeden Finger für sich!) und die Arme 5 Minuten lang mit Seife und warmem Wasser ab. Der Seifenschaum wird in frischem Wasser von Händen und Bürste abgespült (Seife und Sublimat gibt eine Verbindung, die die antiseptische Kraft des Sublimats auf hebt!) und sodann werden die Nägel, Hände und Arme in einer $0,1 \%$ igen Sublimat-oder $3 \%$ igen Carbolsäurelösung 
2 Minuten lang gebürstet. Hat man etwa $500 \mathrm{~g}$ Brennspiritus zur Hand, so lässt sich nach Fürbringer die Desinfektion in 3 Minuten machen, indem man die Hände je eine Minute mit Seife und warmem Wasser, in Spiritus und in $0,2 \%$ iger Sublimat-oder $3 \%$ iger Carbolsäurelösung bürstet.

\section{Desinfektion der Instrumente.}

Katheter und Glasrohr (bei voraussichtlicher operativer Entbindung auch die hierzu nötigen Instrumente) werden in einem durch Deckel versehlossenen Topf (Wasehkessel) 5-15 Minuten lang gekocht und sodann von desinfizierter Hand in einen Topf oder Schüssel mit 3\% iger Carbolsäurelösung hineingelegt. Mit. dem Messglas zu $30 \mathrm{~g}$ lässt sich die für einen Liter der genannten Lösung notwendige Quantität der reinen Carbolsäure genau abmessen. Die Mischung muss vor dem Gebrauch tüchtig umgerührt werden, bis keine Carbolperlen mehr in derselben zu bemerken sind. Der Heberschlauch wird bis zu seinem Gebrauch in der Sublimatlösung aufbewahrt.

Hat man die Instrumente bei einem infektionsverdächtigen Fall gebraucht, so reinige man sie mit warmem Wasser, Seife und Bürste und koche sie sofort aus. Wo das nicht möglich, lege man sie nicht in die Tasche zurück, sondern schlage sie für sich in Papier und koche sie bei sich aus.

Besitzt man einen Trockensterilisator, so geschieht die Desinfektion der Instrumente am einfachsten in der Weise, dass man die in einem Kupfer- oder Nickelinkasten untergebrachten Instrumente durch trockene Hitze sterilisirt.

\section{Desinfektion der Kreissenden.}

Im Prinzip sollte man bei jeder Kreissenden die äusseren Geschlechtsteile desinfizieren.

Gründlich ist dies nur auf dem Querbett möglich. Das Querbett macht man am schnellsten in der Weise zurecht, dass man die beiden Unterarme unter die Kniee der Frau sehiebt, den Steiss bis an den Bettrand zieht, die Füsse auf einen Stuhl stellt und nun noch den Oberkörper der Frau mitsamt einem Kissen so herumdreht, dass die Kreissende nicht mehr schräg, sondern quer liegt. 
Darauf seift man, nachdem man vorher die eigenen Hände noch einmal in der Sublimatlösung übergebürstet hat, zwischen den auf 2 Stühlen stehenden Schenkeln der Frau sitzend, mit der einen Hand die äusseren Geschlechtsteile und ihre Umgebung ab, indem man mit der anderen Hand diese Teile mit $3 \%$ iger Carbolsäurelösung überrieselt. Die Flüssigkeit läuft an der reinen Unterlage in einen ans Bett geschobenen Eimer ab. Den etwa notwendigen Katheterismus nimmt man zweckmässig jetzt vor. Hat bereits eine innere Untersuchung seitens der Hebamme stattgefunden, so schliesst man an die Desinfektion der äusseren Genitalien sofort eine Scheidenausspülung an, um etwa schon eingeführte Infektionskeime noch nachträglich unschädlich zu machen: $\mathrm{Zu}$ diesem Zweck bürstet man seine Hände nochmals in der Sublimatlösung ab, führt Zeige- und Mittelfinger der linken Hand in die Scheide ein und dirigiert mit diesen Fingern das von der rechten Hand gehaltene Rohr in alle Falten der Scheide und ev. auch in den (geöffineten) Muttermund. Die beiden Finger reiben dabei die Scheidenwände sanft ab, um das ihnen anhaftende Sekret zu entfernen. Für diese Ausspülung genügt ein Liter einer $3 \%$ igen Carbolsäurelösung. Hierdurch wird nach $\mathrm{Steffeck}$ die Scheide vollständig keimfrei gemacht. Es folgt nochmaliges Abbürsten beider Hände, Einfettung des nassen Zeigefingers, ev, auch des Mittelfingers der reehten Hand mit Boroglycerin, und nun nimmt man die innere Untersuchung gleichfalls auf dem Querbett vor, welches die innere Untersuchung sehr erleichtert. Besonders über den Stand des Kopfes im Becken gewinnt man bei dieser Untersuchung oft eine ganz andere Anschauung, als bei der Untersuchung auf dem Längsbett. Vor der Untersuchung dart die desinfizierteHand, wenn man die Desinfektion nicht wiederholt, mit nichts mehrin Berührunggebrachtwerden, als mitden Genitalien. Man darf also nicht etwa noch mit den Händen an die eigenen Kleider kommen oder einen Stuhl, die Bettdecke etc. anfassen.

In der Praxis pauperum stösst dieses Vorgehen auf keine Schwierigkeiten, und in der Praxis elegans lässt es sich ebenfalls durchführen, falls man der Kreissenden etwas 
Chloroform gibt. Auch erleichtert dieses Mittel nebenbei noch sehr die Untersuchung. Der straffe Damm Erstgebärender lässt sich in der Narkose mehr nach oben dislocieren und der Finger kann höher hinaufdringen: Manche unglückliche Zange würdevermieden worden sein, wenn vor der Operation die innere Untersuchung anf dem Querbett und in Narkose angestellt worden wäre!

Muss man aus äusseren Gründen von der Narkose und der Desinfektion auf dem Querbett absehen, so kann man sich allenfalls bei einer Kreissenden, die vor oder im Beginn der Geburt eine Mastdarmeingiessung erhalten und dann warm gebadet hat, mit einer blossen Abspülung der äusseren Geschlechtsteile and einer Scheidenausspülung (mit 3\% iger Carbolsäurelösung) auf den Längsbett begnügen. Zu diesem Zweck wird der Kreissenden ein Stechbecken untergeschoben.

Bei der inneren Untersuchung selbst konstatiert man ohne weiteres, ob der Scheideneingang und die Scheide selbst eng oder weit sind. Die Scheide kann durch eine weiche, oft aber anch knollig harte Vertreibung der hinteren Wand verengt sein, die von einer starken Anfüllung des Mastdarms herrührt. In diesem Fall muss durch eine Eingiessung der Mastdarm entleert werden, weil sonst beim Durchschneiden des Kopfes der Koth ausgepresst wird und die Unterlagen, den Damm und die stützende Hand beschmutzt.

Weiter stellt man die Grösse des Muttermundes fest. Man bezeichnet denselben als :

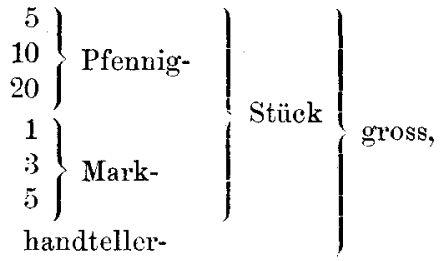

völlig erweitert.

Bei Mehrgebärenden, wo im Anfang der Geburt der äussere Muttermund noch seine quere Gestalt bewahrt, taxiert man auch die Grösse nach der Zahl der Finger, welche man hindurchführen kann. Noch exakter wird die Angabe über die 
Grösse des Muttermundes, wenn man auch die Breite des vorhandenen Saums nach Fingerbreiten bestimmt. Man prüfe ferner die Dicke dieses Saums resp. füble nach, ob der Cervicalkanal noch über den Ansatz der Scheide hinaus erhalten ist (s. Fig. 19). Hierbei werden dem Untersucher eine auffallende Weichheit (bei Plac. praev.) oder Rigidität oder eine carcinomatöse Degeneration des Saumes resp. der Portio nicht entgehen.

Die Angabe der Kreissenden oder Hebamme, dass Wasser abgegangen, ist nicht beweisend dafür, dass der Blasensprung bereits stattgefunden. Es kann sich dabei um Abgang von mütterlichem Urin oder von sogenanntem falschen Wasser, d. h. einer zwischen Chorion und Amnion angesammelten Flüssigkeitsmenge gehandelt haben. Andererseits kann aber auch die Blase (an einer höheren Stelle) bereits gesprungen sein, obgleich sie vor dem Kopf noch intakt erscheint. Fehlt das Vorwasser ganz, so liegt die Blase dem Kopf fest an, man erkennt sie dann aber an ihrer Glätte resp. daran, dass man keine Haare fühlt.

Fine weitere Frag, ist die nach der Finstellung and dem Stand des Kopfĕs im Becken. Steht der Kopf noch über dem Beckeneingang, so kann man das Promontorium mit gestrecktem Finger erreichen und die Conj. diag. messen, steht er mit seinem grössten Umfang im Beckeneingang, so ist das Promontorium nur mit gekrümmtem Finger zu erreichen, also nicht mehr zu messen, steht der Kopł mit seinem grössten Umfang in der Beckenweite, so befindet sich die Leitstelle in (ler Spinallinie, der Verbindungslinie der Spinae ischii. Kann man die Spinae ischii nicht oder nur noch eben erreichen, so steht der Kopf in der Beckenenge und im Beckenausgang dann, wenn man den Finger kaum noch eive kurze Strecke zwischen Kopf und hinterer Vaginalwand in die Höhe schieben kann.

Steht der Kopf trotz lüngerer Dauer der Geburt und kräftiger Wehenthätigkeit noch über dem Beckeneingang, so ist zunächst an ein enges Becken, dann aber auch an eine abnorme Grösse des Kopfes (Hydrocephalus) oder an eine abnorme Einstellung zu denken. Von letzterer kommt bei schädellagen nur die Vorder- und Hinterscheitelbein- 
einstellung, auch vordere und hintere Ohrlage genannt, in Betracht. Bei ersterer verläuft die Pfeilnaht dicht am Promontorium, bei letzterer dicht an oder über der Symphyse. Die Vorderscheitelbeineinstellung mit Tiefstand der grossen Fontanelle machtein plattes Becken höchst. wahrscheinlich. Um letzteres zu diagnosticieren, wird man zunächst das Becken austasten; bei plattem Becken erreicht man schon mit einem Finger das Promontorium, welches bei normalem Becken grade nur mit zwei Fingern zu erreichen ist. Bei allgemein verengtem Becken ist das Prom. mit einem Finger zu erreichen, und ausserdem sind die Seitenwände des Beckens sehr leicht vollständig zu bestreichen. Für die Prognose und Therapie einer Geburt ist es indessen unerlässlich, ausserdem noch eine genaue Beckenmessung rorzunehmen. Man misst also zunächst die Conj. diagonalis (13), darauf den Dia meter spinarum (25) und cristarum (28) und die Conj. externa (20).

Steht der Kopf trotz kräftiger Wehen abnorm lange im Beckenausgang, so handelt es sich meistens um einen abnormen Widerstand seitens der Weichteile, seltener um tiefen Querstand (Verlauf der Pfeilnaht im queren Durchmesser), am seltensten um ein im queren Durchmesser des Beckenausgangs verengtes, also um ein kyphotisches oder Trichterbecken. Die Diagnose des letzteren wird zunächst nur per exclusionem gestellt: Wenn die Weichteile des Beckenbodens nicht abnorm rigide sind, wenn kein tiefer Querstand vorhanden, so muss der Widerstand von Seiten des Beckens ausgehen - weiterhin durch die Messung des queren Durchmessers des Beckenausgangs. Nimmt man diese sowohl nach der Methode von Breisky als auch nach der von Schröder vor, so erhält man recht genaue Resultate. Bei der ersten Methode drückt man die nach aussen gerichteten Knöpfe des Beckenmessers fest gegen die Innenfläche der Tub. ischii. $Z u$ dem erhaltenen Mass muss man $1 / 2-1^{1 / 2} \mathrm{~cm}$ hinzuaddieren. Schröder projiciert die Messpunkte auf die äussere Haut, markiert sich die betreffenden Stellen und misst direkt ihre Entfernung.

Ist der Kopf in günstiger Stellung ins Becken eingetreten, d. h. also mit in der Mitte $z w i s c h e n$ 
Symphyse und Promontorium verlaufeuder Pfeilnaht und gesenkter kleiner Fontanelle, befinden sich Mutter und Kind wolll, so hat der Geburtshelfer ruhig abzuwarten, aber aufmerksam den Geburtsverlauf zu beobachten, um etwaige Storumgen frühzeitig zu erkennen.

Man wird also die Art der Wehenthätigkeit ins Auge fassen und feststellen, wie bäufig die Wehen aufeinander folgen, ob die einzelne Wehe kräftig und ihrer Stärke entsprechend schmerzhaft ist, ob und wann die Bauchpresse in Aktion tritt. Von Zeit zu Zeit wird man nach gehöriger Desinfektion der Hände die innere Untersuchung wiederholen, um festzustellen, ob sich der Muttermund mehr geöffnet hat, der Kopf tiefer, die kleine Fontanelle mehr nach vorn getreten ist.

Nach dem Blasensprung muss man jedenfalls innerlich untersuchen, um einmal die Stellung des Schädels aufs Genaueste festzustellen, was vor dem Blasensprung wegen der Gefahr der Blasensprengung, längere Zeit nach demselben wegen der Kopfgeschwulst oft schwierig ist - und andererseits nachzufühlen, ob nicht etw a di e $\mathrm{Nab}$ a lschnur oder der Arm des Kindes neben dem Kopf vorgefallen sind.

Sind die Wehen schwach, so darf dic Kreissende, wemn sie es wünscht, auch nach dem Blasenspruvg aufstehen und umhergehen, vorausgesetzt, dass der Kopf bereits fest ins Becken eingetreten ist. Die Wehen werden danach hin und wieder kräftiger. Das willkürliche Mitpressen darf man erst dann gestatten, wenn der Kopf nach völliger Erweiterung des Muttermundes tief in der Scheide steht, also erst gegen Ende der Austreibungsperiode. Manchen Frauen ist es dann angenehm, wenn sie die Füsse gegen einen ins Bett gesetzten Schemel stemmen und sich mit den Händen an irgend einer Handhabe festhalten können. $\mathrm{Zu}$ dieser Zeit stellt sich meistens starker Drang zum Stuhlgang ein. Hat man vorher den Mastdarm entleeren lassen, so ist dem Verlangen. der Frau nach dem Stechbecken nicht nachzugeben.

Dammschutz: Kommt der Kopf zum Einschneiden, und wird der Damm stärker gespannt, so lagere man die Kreissende 
auf die Seite, entferne alle Handhaben und untersage das Mitpressen, resp. lasse die Kreissende rasch ein- und ausathmen. Die vom Bauch her zwischen den Schenkeln der Kreissenden durchgeführte Hand wird auf den geborenen Teil des Kopfes gelegt und hält sodann während der Wehe den Kopf zurüek, falls bei derselben der Damm allzustark verlängert und gespannt wird, während Zeigefinger und Daumen der unteren Hand dem Frenulum labiorum dicht anliegen und dasselbe durch eoncentrisches Zusammenziehen der Seitenpartien des Damms zu entspannen suchen. Der eigentliche Druck mit der yanzen Hohlhand auf den Damm sollte vermieden werden, da grade er oft das Aufplatzen des Damms bewirkt. Ist der Kopf ungefähr bis zur grossen Fontanelle geboren, so streife man in der Wehenpause den Damm nach hinten hin zurück oder lasse die Kreissende den Kopf ausserhalb der Wehe herauspressen oder endlich mache den sehr zweckmässigen, von $01 \mathrm{~s}-$ hausen in die Praxis eingeführten Ritgen'schen Handgriff, d. h. drücke die Stirn und dann das Gesicht durch 2 in's Rectum eingeführte Finger heraus. Der Handgriff hat nur das Unangenehme, dass man nach seiner Anwendung sich wieder gründlich desinficiren muss. Manchmal gelingt es, die Stirn vom Hinterdamm, dem hinter dem Anus gelegenen Theil des Beckenbodens, aus herauszudrücken (Ritg en, Fehling.)

Nimmt der Damm eine weissliche Färbung an, so steht er im Begriff zu reissen. Während man den Kopf von der Hebamme zurückhalten lässt, macht man unter Leitung von Zeige- und Mittelfinger der linken Hand mit einer Cowperschen Scheere einen Schnitt in die seitliche Partie des Frenulum in der Richtung auf das entsprechende Tuber ischii. Der Schnitt durchtrennt zunächst die Haut, in der Wunde erscheint dann der scharfe Saum der Fascie, in welchen man mit einem zweiten Scheerenschlag einschneidet. Nun ziehe man in der Wehenpause den Damm vorsichtig nach hinten. Macht dies Schwierigkeiten, so vertiefe man die Incision lieber, als dass man eine zweite anlegt (Credé, Fehling). Man schneide also ev. noch in der Constrictor cunni hinein. Durch die gleichmässige Retraction der beiden Wundränder entsteht Fig. 25. Die Wunde muss stets wieder vereinigt werden, weil sonst 
eine Verlängerung der Vulva fast um das Doppelte der ursprünglichen Länge der Incision $(1-3 \mathrm{~cm})$ entsteht. Oft genügt zur Wiedervereinigung eine einzige von $\mathrm{c}$ nach $\mathrm{b}$ geführte Naht.

Nach Geburt des Kopfes fühle man nach, ob die Nabelschuur um den Hals geschlungen ist. Ist dies der Fall, so lockere man die Schlinge und streife sie über den Kopf resp. die Schultern zurück. Sodann reinige man sofort mit einem. reinen in abgekochtes Wasser getauchten Leinwandläppchen. sehr sorgfältig die Augen des Kindes. Es folgt jetzt die Fixpression des Rumpfes durch kräftigen nach abwärts gerichteten Druck auf den Fundus uteri. Zögert trotzdem der Austritt, so extrahire man vorsichtig, indem man in die hintere Achselhöhle vom Rücken her einen Zeigefinger einführt. Ist die hintere Schulter geboren, so entwickle man die vordere durch Senken des Kopfes.

Ist das Kind nicht asphyctisch, schreit und athmet es also gleich kräftig, so warte man mit der Abnabelung, bis die Nabelschnur nur noch schwach pulsirt. Zwischen der Unterbindung und dem Nabel muss so viel Raum bleiben, dass man bei Nachblutungen eine zweite Ligatur anlegen kann, also etwa $4 \mathrm{~cm}$. Nach der Placenta hin legt man eine 2. Ligatur, einmal der Reinlichkeit wegen, und weil die blutstrotzende Placenta sich leichter löst.

\section{Behandlung der Nachgeburtsperiode.}

Die Placenta wird in Deutschland allgemein durch den Credé's chen Handgriffentfernt. Differenzen bestehen nur in der Zeit seiner Anwendung. Man wartet am besten die spontane Lösung der Placenta und der Eihäute ab. Dieselbe erfolgt durch die Nachgeburtswehen innerhalb der ersten halben Stunde nach der Geburt und markirt sich durch das Vorrücken der Nabelschmur (Ahlfeld) und durch die feste und andauernde Contraction bezw. Retraction des Uteruskörpers, welcher nach Ausstossung der Placenta in den Durchschnittsschlauch häufig über den Nabel ansteigt (Schröder, E. $\operatorname{Coh} n$ ).

Während es das Verdienst von Credé ist, durch seinen Handgriff die alte Methode der Herausbeförderung der Placenta 
durch Zug an der Nabelschnur beseitigt zu haben, ist es das Verdienst von Dohrn und Ahlfeld, dass sie dafür eintraten, bei normalen Geburten die Lösung der Placenta nicht durch Reiben des Uterus, wie Credé es wollte, zu beschleunigen und die Anwendung der Expression zeitlich weiter hinauszuschieben.

Den Handgriff nehme man $1 / 2-1$ Stundenach der Geburt nur während einer Wehe und bei leerer Harnblase vor, indem man den Fundus mit der vollen Hand umgreift (4 Finger an der hinteren, Daumen an der vorderen Wand) und ihn gegen die Höhlung des Kreuzbeins nach hinten und abwärts drïckt. Die andere Hand ergreife die in der Schamspalte sichtbar werdende Placenta und drehe sie mehrmals herum, um die Eihäute zu einem Strang aufzuwickeln und so ein Abreissen desselben zu verhüten. Gelingt der Handgriff nicht gleich, so warte man die nächste Wehe ab und nehme ev. die 2. Hand zur Hülfe. (Die Heb. muss hierbei ein zu schnelles Hervorschiessen der Placenta verhindern, da hierbei die Eihäute leicht abreissen.)

\section{Behandlung der Clitoris- und Dammrisse.}

Nach der Geburt reinige man die äusseren Geschlechtstheile mittels eines Bausches in 3\%ige Carbolsäure getauchter Sali-

Fig. 1.

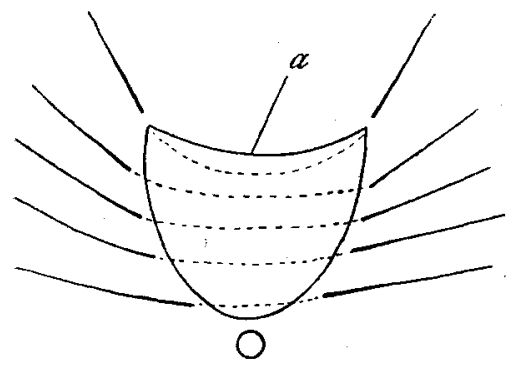
cylwatte und sehe sich nach etwaigenVerletzungen um. Risse in der Gegend der Clitoris müssen wegen der starken Blutung, Dammrisse, um für die Zukunft einen Vorfall der vorderen Scheidenwand zu vermeiden, genäht werden. Es ist principiell gewiss richtig, jeden Dammriss von $1 \mathrm{~cm}$ Länge an durch die Naht zu schliessen. Bis man die Vorbereitungen zu derselben getroffen, muss man oft wegen stärkerer Blutung die provi- 
Fig. 2.

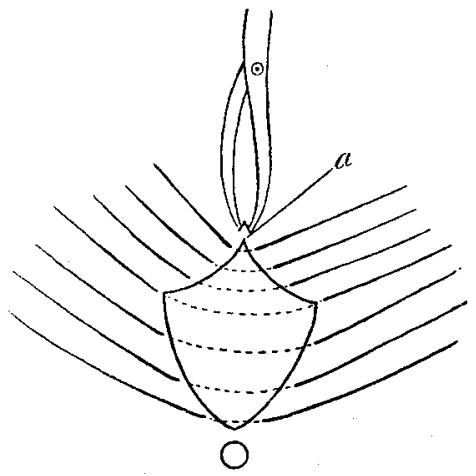

sorische Tamponade desselben mit einem Streifen Jodoformgaze ausführen. Man vergesse nicht. dass. der Dammriss sich gewöhnlich in einen oder zwei Scheidenrisse seitlich neben der Columna rugarum post. hinein fortsetzt (Freund, s. Fig. 3 bis 6). Diese müssen zunächst sorgfältig vereinigt werden, indem man ev. ihre Spitze mit einer $\mathrm{Ku}$ gelzange hervorzieht. Die

Vernähung eines Dammrisses in Seitenlage lässt diese Scheidenrisse unvereinigt.

Man kann mit der Naht des Dammrisses 12-24 Stunden warten. Man soll daher nicht bei Nacht nähen, sondern provisorisch mit Jodoformgaze tamponiren. Die Narkose ist im Interesse einer exacten $\mathrm{Naht}$ sehr zu empfehlen. Hatte Verf.

Fig. 4.

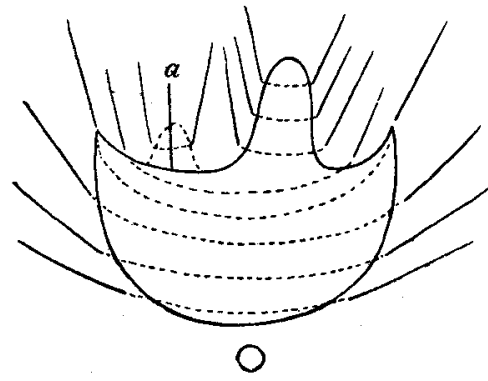

Fig. 3.

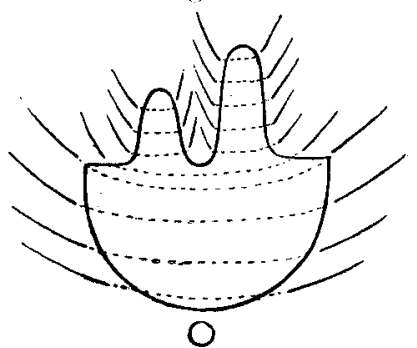

die Geburt selbst nicht geleitet, so nimmt er vor der Naht stets eine Scheiden- und Uterusausspülung mit $3 \%$ iger Carbolsäurelösung vor, um etwaige eingedrungene Infectionskeime, welche die prima intentio vereiteln würden, noch nachträglich zu vernichten. Die äusseren Geschlechtsteile 
werden rasirt, die Wunde während der Naht mit Sublimat $1: 5000$ überspült. Benutzt man Seide, so sterilisire man diese

Fig. 5.

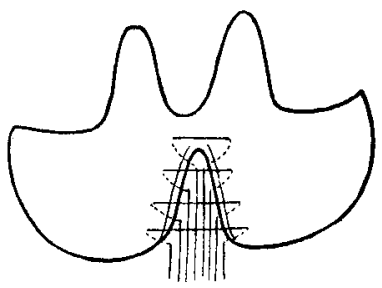

Fig 6.

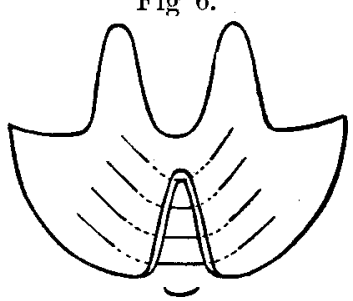

unmittelbar vor ihrem Gebrauch durch 5 Minuten langes Auskochen. Einen Dammriss 3. Grades verwandelt man zunächst Fig. 7 .

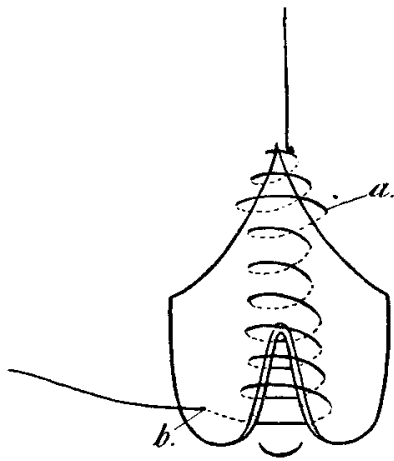
durch von der Wunde aus angelegte versenkte Catgutnähte, die die Schleimhaut des Darms nicht mitfassen, in einen Dammriss 2. Grades (Fig. 6), den man, wie in Fig. 1-4 angedeutet, vernäht. Wer in der fortlaufenden Catgutnaht Uebung hat, wählt zweckmässig diese (Fig. 7-9). Beim Dammriss 3. Grades braucht man mindestens 2 , meistens 3 Nahtreihen*). Die Nachbehandlung bei allen (genähten oder nicht genähten) Wunden der äusseren Genitalien besteht in der Praxis nach dem Vor-

*) Bezüglich der Figuren ist noch zu bemerken, dass Fig. 1 einen Dammriss 2. Grades ohne Scheidenriss, Fig. 2 denselben darstellt, bei dem nur Punkt a durch eine Kugelzange fixirt ist; Fig. 4 zeigt einen Dammriss 2. Grades mit einseitigem Scheidenriss. Bei a ist der Wundrand der exacten Vernähung halber zu einem Plissé (Küstner) erhoben und mit einer Naht versorgt. Fig. 5 (Dammriss 3. Grades mit doppeltem Scheidenriss), zeigt die Art der Nahtanlegung vom Mastdarm aus. Diese muss man wählen, falls man mit Seide oder Silkworm näht. 
schlag von Fitseh am zweckmässigsten in oft gewechselten. Umschlägen von Wundwatte oder ausgekochter Leinwand, die mit einer $0,05 \%$ gen Sublimatlösung getränkt sind.

Fig. 8 .

Fig. 9.*)

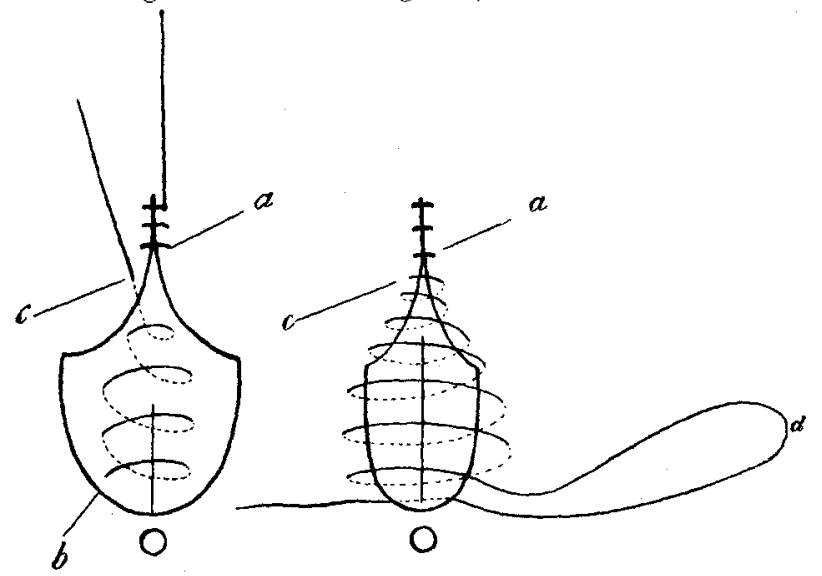

Für weichen Stuhlgang ist am 3. oder 4. Tag zu sorgen. Die Diät muss eine hauptsächlich flüssige sein. Heilt der Riss nicht p. p., so nehme man vom 8. Tag an, nach Anfrischung der Wundränder und Ablratzen der Granulationen die Secundärnaht vor. Die Erfolge derselben, die in dieser Weise ausgeführt, eine frühzeitig gemachte plastische Operation darstellt, müssen stets gute sein, falls man unter antiseptischen Cautelen operirt und das richtige Nahtmaterial wählt. Nur bei Dammrissen 3. Grades empfiehlt Verf. die Schliessung des Mastdarmrisses durch eine fortlaufende versenkte Catgutnaht, für alle Dammrisse 2. und 1. Grades dagegen die Seidenknopfnaht, weil die wenigen Knopfnähte, die ausserdem ihren festen Halt an der Haut finden, das brüchige Gewebe nicht so leicht durchschneiden, wie die zahlreichen Schlingen der fortlaufenden Naht.

*) Bei $d$ wird die Schlinge durchschnitten, der untere Faden geknüpft und dann ein Fadevende dfsselben mit dem oberen Faden zusammengeknotet. 


\section{Behandlung der 3. und 4. Schädellagen (Vorderhauptslagen) und des Tiefstandes der grossen Fontanelle.}

Man lagere die Kreissende auf die Seite der kleinen Fontanelle (A n m.: Man lagert die Frau stets auf die Seite, wo der Theil steht, dessen Tiefertreten man wünscht!). Dieselbe wendet sich manchmal noch im Beckenausgang von hinten nach vorn. Tritt bei nach vorn gerichteter grosser Fontanelle eine Indication zur Beendigung der Geburt auf, so lege man die Zange so an, dass die grosse Fontanelle nach vorn kommt. Alle künstlichen Drehungen des Kopfes mit der Zange, die die kleine Fontanelle nach vorn bringen sollen, sind obsolet.

Steht die grosse Fontanelle seitlich aber tiefer als die kleine - dies bezeichnet man nicht als Vorderhauptslage, sondern als Tiefstand der grossen Fontanelle -- so muss man zwei Dinge auseinderhalten:

1) Der Kopf steht noch über dem Becken.

Verläuft dabei die Pfeilnaht dicht am Promontorium, so handelt es sich um ein plattes Becken, bei welchem man ev. die prophylactische Wendung vornehmen wird. Verhält man sich dagegen exspectativ, so wird man in diesem Fall die Kreissende so lange auf die Seite der grossen Fontanelle lagern, bis der verengte Beckeneingang überwunden ist (das schmälere Vorderhaupt passirt dabei die verengte Conjugata). Ist dies geschehen, so lagere man die Kreissende auf die andere Seite, um das Tiefer- und Nachvornetreten der kleinen Fontanelle zu begünstigen.

2) Der Kopf hat den Beckeneingang passirt.

Ist die Beendigung der Geburt indicirt, so versuche man zunächst durch kräftigen Druck mit beiden dicht über der Symphyse auf den Kopf aufgelegten Händen denselben tiefer ins Becken hineinzupressen. Häufig tritt hierbei die kleine Fontanelle tiefer und nach vorn. Ist dies nicht der Fall, so lege man auch hier die Zange so an, dass die grosse Fontanelle sich nach vorne dreht.

Sorgfältiger Dammschutz ist sowohl bei den Vorderhauptsals auch den Stirn- und Gesichtslagen nothwendig! 


\section{Behandlung der Stirn- und Gesichtslagen.}

Nur bei Komplikation dieser Lagen mit plattem Becken oder Vorfall der Nabelschnur oder eines Armes nehme man vornherein die Wendung auf einen Fuss vor. Im Uebrigen verhalte man sich exspectativ und suche nur durch Lagerung auf der Seite der kleinen Fontanelle eine Schädellage herzustellen. Tritt der Kopf trotzdem in einer der genanuten Lagen ins Becken ein, so lagere man die Kreissende auf die Seite des Kinns, damit aus der Stirnlage eine Gesichtslage wird, resp. bei Gesichtslage das Kinn tiefer und nach vorne tritt.

Sehr selten sind die Fälle, wo das Kinn auch noch im Beckenausgang hinten oder seitlich steht. Tritt dam eine Gefahr für die Mutter auf, so bleibt bei nach hinten gerichtetem Kinn nur die Perforation übrig. Zange wäre ein Kunstfehler. Bei nach der Seite gerichtetem Kinn ist zwar ein Zangenversuch erlaubt. In den Fällen des Verf., in denen es sich stets um sehr grosse exquisit dolichocephale Köpfe handelte, gelang derselbe allerdings nie. Unter 18 Fällen, die Salomon zusammengestellt hat, wurde mit der Zange nur einmal ein lebendes Kind entwickelt. Diese sehr seltenen Fälle geben keinen Grund, prinzipiell bei Gesichts - und Stirnlagen die Wendung zu machen. Man vergesse nicht, dass infolge der Wendung (wenn sie nicht von sehr geübter Hand ausgeführt wird) eine viel grössere Zahl von Kindern zu Grunde geht, als beim Abwarten. Was die manuelle Umwandlung dieser Lagen in Schädellagen durch kombinierte Handgriffe von innen und aussen anbelangt, so hat Verf. von derselben bei grossen dolichocephalen Köpfen nie einen Erfolg gesehen, in den übrigen Fällen aber nie ein Bedürfnis nach derselben empfunden.

\section{Behandlung der Geburt bei Beckenendlagen.}

Wird beiBeckenendlagen nach Geburtdes Nabels das Kind nicht binnen wenigen Minuten geboren, so erstickt es infolge der Kompression der Nabelsehnur zwischen Becken und kindlichem Rumpf resp. Kopf. Eine solche Geburtsverzögerung tritt besonders leicht ein bei I p. mit straffer, enger Scheide, bei engem Becken,

Dührssen, Geburtshülfliches Vademecum. 
bei grossem Kind, bei vollkommener Fusslage, da die Füsse und schmalen Hüften die Weichteile für den raschen Durchtritt des Kopfes nicht genügend erweitern, - endlich falls man vor Geburt des Nabels an den geborenen Teilen gezogen hat.

Mit Ausnahme von Steisslagen, die mit Nabelschnurvorfall und engem Becken kompliciert sind, und bei denen man prophylaktisch einen Fuss herunterholt, um das Kind bei Gefährdung seines Lebens leicht extrahieren zu können - verhalte man sich bei Beckenendlagen, bei denen weder Mutter noch Kind in Gefahr sind, streng exspektativ bis zur Geburt des Nabels. Zieht man früher an den geborenen Teilen, so können sich die Arme in die Höhe schlagen, das Kinn sich von der Brust entfernen - Umstände, die die schnelle Entwicklung der Arme und des Kopfes sehr erschweren oder unmöglich machen. Man untersage der Kreissenden das Mitpressen, instruiere sie aber, dass sie später (nach Geburt des Steisses) auf gegebenes Kommando aus allen Kräften nach unten drängen müsse. Auch belehre man, da später zu langen Auseinandersetzungen keine Zeit ist, die Hebamme, dass sie nach Geburt des Steisses auf gegebenes Kommando den Fundus mit beiden Händen umfasse und ibn kräftig nach abwärts drücke, dass sie aber mit diesem Druck aufhöre, sobald die Arme gelöst sind, und dann beide Hände unmittelbar über der Symphyse auf den Kopf setze und diesen mit aller Kraft abwärts, ins Becken hinein, drücke. Man lege sich ferner alle Sachen zur Wiederbelebung scheintoter Kinder zurecht und erinnere an das Badewasser, welches man bereits beim Einsehneiden des Steisses ins Zimmer bringen lässt.

Mehrgebärende bringe man, wenn der Steiss in die Scheide hineintritt, Erstgebärende, wenn er zum Einschneiden kommt, auf's Querbett, da nur so die ev. notwendige Hülfe geleistet werden kann.

Vielfach wird nnmittelbar nach Geburt des Steisses unter Mitpressen der Kreissenden und unter Anwendung der eben beschriebenen (Kristeller'schen) Expression der übrige Teil des Kindes geboren. Ist dies nicht der Fall, so schreite der Geburtshelfer, der sich selbstverständlich vorher erst gründlich desinficiert hat, zur 


\section{Lösung der Arme und des Kopfes.}

$\mathrm{Zu}$ dem Ende wird zunächst die straffe Nabelschnur etwas gelockert, sodann umfassen beide Hände den in eine warme Windel gehüllten Steiss in der Weise, dass die Daumen auf den Hinterbacken, die Zeigefinger auf den Darmbeinkämmen, die übrigen Finger an den Obersehenkeln liegen. Sind die Füsse in die Höhe geschlagen, so setze man beide Zeigefinger in die Hüftbeugen ein und ziehe nun den Rumpf stark nach abwärts und unter Heben und Senken des Steisses bis zum Angulus scapulae hervor, wobei der Rücken nach der Seite gekehrt bleiben muss. Sodann fasst man mit der Gabel der einen Hand die Füsse des Kindes und legt sie in die Hüftbeuge der Mutter, nach welcher der Bauch des Kindes gerichtet ist, geht mit Zeige- und Mittelfinger der anderen Hand vom Rücken des Kindes her an dem hinteren Arm bis zum Ellenbogengelenk hin und drücke die Arme nach der Bauchscite dès Kindes hin herab. Dann fasse man den Rumpf möglichst hoch in der Weise, dass die Daumen auf dem Rücken, die übrigen Finger am Thorax liegen und dreht das Kind so herum, dass der Rücken vorne an der Symphyșe vorbeigeht. I)ie Lösung des zweiten hierdurch nach hinten gebrachten Armes geschieht in derselben Weise, wie die des ersten. Man gehe nun mit Zeige**) und Mittelfinger derjenigen Hand, nach welcher der Bauch des Kindes zuletzt gerichtet war, in den Mund des Kindes ein (derselbe steht bei Hochstand des Kopfes mehr seitlich, bei Tiefstand hinten!), setze die Finger auf die Kieferränder, und ziehe das Kinn herab, indem die zweite Hand den Kopf von aussen umfasst und ihn ins Becken lineindrückt (Wi gand-Martin-Winckel'scher Handgriff). Gelingt dieser Handgriff nicht, so ziehe man mit der zweiten Hand am Nacken des Kindes (Veit'scher Handgriff), während die Hebamme den Druck von aussen ausführt.

Misslingt dies ebenfalls, so nehme man selbst den äusseren Druck mit beiden Händen vor. Hierdurch ganz allein habe ich mehrmals den Kopf noch durch ein plattes Becken hin-

*) Man kann auch den Zeigefinger allein einführen. Dieser muss aber dann mit seiner Spitze den Zungengrund erreichen. 
durchgebracht, nachdem die übrigen Methoden erfolglos geblieben waren.

Bekommt man den Kopf bei sachgcmässer Ausführung dieser 3 Methoden überhaupt nicht heraus, so stehe man nach einigen Minuten vou ihrer Fortsetzung ab. In diesen Füllen kam es sich bei weitem Becken und völlig erweitertem Muttermund nur um einen abnorm grossen Kopt resp. um einen Hydrocephalus handeln. Hier perforire man den nachfolgenden Kopf.

Mit Recht betont $Z$ weifel, dass es ein Kunstfehler sei, wenn bei der beschriebenen Operation, die man streng von der eigentlichen Extraction am Fuss oder Steiss trenuen muss, dem Geburtshelfer das vorher noch lebensfrisehe Kind abstirbt, vorausgesetzt, dass eben nicht vorher gezogen, dass kein enges Becken, kein mangelhaft eröffneter Muttermund, kein abnorm grosses Kind vorhanden war. Und doch - wie häufig kommt dieses Ereigniss vor! Die beschriebene Operation ist allerdings, meiner Ansicht nach, die allerschwierigste, weil eine ganze Reihe von Handgriffen rasch hintereinander gemacht werden müssen. Fleissige Einübung dersellyen am Phantom ist unerlässlich.

Die Prognose der Beckenendlagen ist, falls zur Rettung des Kindes keine gewaltsamen Eingriffe stattfinden, für die Mutter nicht schlechter als die der Schädellagen. Die Prognose für die Kinder hängt ganz und gar von der Geschicklichkeit des Geburtshelfers ab.

\section{Behandlung der Querlagen.}

Bei Querlage ist nach dem Blasensprung die sofortige Wendung auf den Fuss zu machen. Ist die Wendung nicht mehr möglich, resp. für die Mutter zu gefährlich, (so nehme man die Zerstückelung des Kindes vor (s. Wendung). Nur bei Fehlgeburten kann man sich exspectativ verhalten. Zieht sich aber bei ihnen die Geburt in die Länge, so wird man durch Zug am Arm den Vorgang der Selbstentwickelung resp. der Geburt conduplicato corpore beschleunigen. 